\title{
Readiness of Government Organizations for Cloud-Computing Age: An Empirical Evidence from Jordan
}

\author{
Ammar Salamh Mujali Al-rawahna, ${ }^{1, *}$, Chung-Wen Hung ${ }^{1}$, Shih-Chih Chen ${ }^{2}$ \\ ${ }^{1}$ Southern Taiwan University of Science and Technology, Tainan, Taiwan, \\ No. 1, Nan-Tai Street, Yungkang Dist., Tainan City 710, Taiwan \\ ${ }^{2}$ National Kaohsiung University of Science and Technology, Kaohsiung, Taiwan, \\ No.1, University Rd., Yanchao Dist., Kaohsiung City 824, Taiwan \\ *Corresponding author: Da21G204@stust.edu.tw
}

Received August 05, 2018; Revised September 07, 2018; Accepted September 19, 2018

\begin{abstract}
The aim of this study is examining the readiness of the Jordanian government sector to adopt cloudcomputing. The study empirically applied the TOE research framework that enables targeting IT managers and IT specialists in several Jordanian organization, where 132 respondents distributed on three sub-groups were targeted. The obtained data were analyzed using Partial Least Square because of the small sample size. The result indicated that top management support, organization capability and government policy reflect the organizational readiness of government organizations to adopt cloud-computing. The multigroup analysis results revealed that Although the size of organizations varies, they have similar drivers of cloud-computing adoption. The IT-related experience has significant negative effect on intention of adoption while cloud knowledge insignificant. The study demonstrated the importance of the pre-adoption step that decision-makers should be aware of organization readiness before taking the decision of adoption, otherwise the organization will fall in cloud-computing trap. The study yielded some practical evidence, for instance, government organizational readiness will help in adopting cloud-computing appropriately to uptake its advantages; IT skills and robust IT infrastructures are both essential pillars for government organization readiness; top management support positively influences the organizational readiness and this an evidence for responsibility of top managers about success of adoption new technology; supportive government policy is substantial issue to strength government organization readiness and persuade government agencies' managers approve transforming process from old IT model into cloud-computing and allocate essential resources.
\end{abstract}

Keywords: organizational readiness, cloud computing adoption, government cloud computing

Cite This Article: Ammar Salamh Mujali Al-rawahna, Chung-Wen Hung, and Shih-Chih Chen, "Readiness of Government Organizations for Cloud-Computing Age: An Empirical Evidence from Jordan." Journal of Business and Management Sciences, vol. 6, no. 4 (2018): 152-162. doi: 10.12691/jbms-6-4-3.

\section{Introduction}

A new era of e-government is unfolding, positioning cloud-computing (CC) as a key delivery model for the electronic services in government domain. CC refers to both the applications delivered as services over the Internet and systems software in the data centers that provide these services and the hardware services as well [1]. This technology provides organizations with opportunities to manage their IT expenditure on an ongoing basis and access a modernist IT resources to provide and manage innovate services continually [2]. The widespread definition of National Institute of Standards and Technology (NIST) described CC as a model for enabling ubiquitous, convenient, on-demand network access to a shared pool of configurable computing resources (e.g. networks, servers, storage, applications, and services) that can be rapidly provisioned and released with minimal management effort or service provider interaction [3]. Government cloud services are a new development at the intersection between e-government and CC which holds the promise to render government service more effective and efficient [4]. Organizations move toward CC may gain benefits such as cost saving, efficiency improving, agility enhancing, flexibility and scalability of services, and environmental sustainability [5].

However, CC presents entirely new challenges and obstacles, particularly in countries coping with limited technical expertise, bandwidth, and IT resources [6]. IT managers of government should have the ability to identify opportunities of CC, and implement them within the governmental IT and policy structures without exposing the departments to unexpected risks [7]. A few IT researchers and practitioners questioned the validity of CC as a competitive alternative over the traditional IT model to 
enhance e-government services [8]. Thus, the investigation in the possibility of $\mathrm{CC}$ to overcome e-government barriers, especially Lack of IT infrastructure is one of the major barriers to success of e-government [9]. The adoption of CC will lead to considerable organizational change that will affect organizations' work style. Governments need to understand the critical factors to adopt CC in organizational manner. This will help to take the informed decision and startup strategy to adopt CC. The early adoption of CC has raised concerns regarding to success this new IT service model. This will lead to question if government organizations ready to embrace the advantages of CC. Where a few studies that highlighted the adoption of CC from an organizational perspective [10]. The challenges and opportunities are different regarding to the organization type and its environment. Managerial IT capabilities are critical to the organization realizing the benefits of CC since they represent the accumulated training and experience, which needed to implement cloud services [11]. The important steps government leaders should take before migrating to cloud are to make organizational assessment where managers should conduct an assessment of their present needs, structure, capacity utilization; and cloud-readiness assessment to determine where cloud can-and cannot-be used as part of your organization's overall it portfolio [12]. UN's report of cloud-computing economy in developing countries advised policymakers at national level to consider following issues in order to translate the potential advantages of CC into tangible development gains: (a) assess the cloud-readiness of the country; (b) develop a national cloud strategy; (c) address the infrastructure challenge; (d) address relevant legal and regulatory issues related to cloud adoption; and (e) Address the need for human resources [13]. Therefore, the main goal of this study is to address most of these issues in order to help developing countries like Jordan taking informed decision toward CC adoption. The essential question of this study about the readiness of government agencies to adopt CC based on perspectives of IT managers and IT specialists.

The rest of this study is organized as follows. Section 2 proposes the CC concept and government initiatives to adopt CC. Section 3 is to develop the research model and hypotheses. Section 4 shows our data analysis procedures and results based on Partial Least Square (PLS). Section 5 discusses the results and the managerial implications based on the hypotheses testing results and previous studies. Finally, the last section presents the conclusions and the proposed theoretical research and practice, as well as future research direction and limitations of this study.

\section{Literature Review}

\subsection{Cloud Services Models}

Most of CC providers such as (Oracle, Microsoft, Amazon, IBM and Google) categorize their services into three major models described as below:

- Software as a Service (SaaS): Applications delivered as a service to end-users typically through a Web browser. In the SaaS model, providers install and operate application software in the cloud, and users access the software from cloud clients without any effort to manage the cloud infrastructure and platform where the application runs [14]. SaaS could provide many benefits for public sector organizations such as cost-savings and better cost control, companied with risks related to difficulty of control IT function [15].

- Platform as a Service (PaaS): An application development and deployment platform delivered as a service to developers who use the platform to build, deploy and manage SaaS applications [16]. Microsoft's Azure and Google's App Engine are fame Platform as a Service. The platform typically includes databases, middleware and development tools, all delivered as a service via the Internet. The service provider manages the cloud infrastructure, operating systems, and the enabling software [17]. PaaS allows customer to create applications using software components that are provisioned and controlled by vendor. PaaS is highly scalable, it facilitates the deployment of applications without the cost and complexity of buying and managing the underlying hardware and software layers [15]. However, the consumer not responsible to manage or control the underlying cloud infrastructure, network, servers, operating systems, or storage, but has control over the deployed applications and, possibly, application hosting environment configurations $[15,18]$.

- Infrastructure as a Service (IaaS): through virtualization, the provider is capable of splitting, assigning, and dynamically resizing the cloud resources including processing, storage, networks, and other fundamental computing resources to build virtualized systems to deliver as a service on-demand based on customer request [18]. The consumer not responsible to manage or control the underlying cloud infrastructure but has control over operating systems, storage, and deployed applications; and possibly limited control of selected networking components (e.g., host firewalls) [19]. IaaS allows organizations to increase or decrease the number of virtual machines running depending on their workload to promote efficiency in the use of IT resources like Amazon's Elastic Compute Cloud (EC2) [20].

\subsection{Deployment Models}

Adopters of CC services can choose the required and needed deployment models among the following:

- Private Cloud: this model is for exclusive use by a single organization and typically controlled, managed and hosted in private data centers that may exist on-site or off-site. This model can be managed by organization itself or a third party [19]. Therefore, the main reason that makes organizations with sensitive information preferred private cloud over a public cloud is usually security: In the private cloud, control over the data remains with customer [21].

- Public Cloud: this model is substantial deployments of hardware and software for use by multiple organizations (tenants) on a shared basis and hosted and managed by a third-party service provider [22]. Cloud providers should ensure continuous the operations and notifying the customer if any service disruption occurred. In some cases, cloud providers 
did not inform the customer for example Amazon EC2 in 2012 when a significant portion of the web disrupted without informed the customers [21,22]. The drawbacks for government using public cloud are data security concerns including data loss and conflicts concerning legal and ethical issues [12].

- Community Cloud: The infrastructure of community cloud is managed for exclusive use by a group of related organizations who wish to make use of a common cloud computing environment, and have shared concerns (e.g., mission, security requirements, policy, and compliance considerations) [23]. Community clouds may be owned, managed, and operated by one or more of the organizations in the community, a third party, or some collection of them [24]. Cost saving of community clouds can come from sharing resources by different institutions and from unifying the purchase, operation and maintenance of hardware and software packages scattered across institutions [25]. Therefore, A community cloud can constitute the optimal venue to provide a distributed environment in which to create a communication platform for performing government incorporated operations.

- Hybrid Cloud: A hybrid cloud combines multiple clouds (private, community of public) where those clouds retain their original features, but are bound together as a unit [23]. For instance, organization could adopt both private and public clouds according to the services types and data sensitivity in order to take advantages of both, such as to reduce costs whilst maintaining privacy and data security. The following table summarizes the deployment models [26].

Table 1. Cloud-Computing Deployment Models

\begin{tabular}{|c|c|c|c|c|c|}
\hline Cloud Type & Description & Admin & Cost & Security & Control \\
\hline Public & $\begin{array}{l}\text { The services are available to the general public and is owned by a } \\
\text { cloud service vendor. }\end{array}$ & Vendor & Low & Low & Lower \\
\hline Community & $\begin{array}{l}\text { The services are shared by several organizations within a specific } \\
\text { community that shares a common mission or set of concerns. }\end{array}$ & $\begin{array}{l}\text { Multiple tenants } \\
\text { or third party }\end{array}$ & Low & Low & Lower \\
\hline Private & $\begin{array}{l}\text { The services are provided solely for a single organization and is } \\
\text { maintained by internal data centers or third parties. }\end{array}$ & $\begin{array}{l}\text { Tenant or third } \\
\text { party }\end{array}$ & High & High & High \\
\hline Hybrid & The services are combination of two or more cloud models. & $\begin{array}{l}\text { Tenant and } \\
\text { vendor }\end{array}$ & \multicolumn{3}{|c|}{$\begin{array}{l}\text { Cost, security and control depend on the } \\
\text { adopted type }\end{array}$} \\
\hline
\end{tabular}

\subsection{Technology-organization-environment (TOE) Framework}

Tornatzky and Fleischer developed TOE framework to explain three elements that influence the decision of adoption new technology at organization-level [27]. These three elements are the technological context, the organizational context, and the environmental context [28]. However, technological context includes all of the technologies that are relevant to the firm - both technologies that are already existed at firm as well as those that are available in the marketplace but not currently in use. The organizational context refers to the characteristics and resources of the firm, including linking structures between employees, internal communication processes, firm size, and the amount of slack resources. The environmental context includes the structure of the industry, availability of technology providers, competitors and the regulatory environment. Regarding to innovation type, economic sectors and different national/cultural contexts, the factors that influence the adoption will be different slightly or totally. Compared to other dimensions, the organizational dimension is pivotal factor which is a combination of organization structure, firm size, centralization, and complexity in management structure [29]. TOE is broadly applicable for exploring the organization-level adopt of different novel IT innovations in qualitative research [30]. This research focused on organizational aspects of government agencies therefore TOE is sufficient to define the research model.

\subsection{Previous Studies}

Serval studies constructed their frameworks based on TOE mainly [31-38]. Some other studies defined the model based on combination of TOE and Diffusion of Innovation Theory (DOI) such as $[10,39]$. In these studies the most dominant organizational factors found to affect CC adoption are technology competency and top management support. Mohammed, Ibrahim, and Ithnin [40], defined organization readiness using similar terms for instance IT skills, IT infrastructures and IT policy. Some studies used technological readiness to reflect technological dimension, top management support to reflect organizational dimension and regulatory support explained the environment factors [10]. Similarly, technology competence and top management support were employed to define the organizational context [14]. Lian, Yen and Wang [34] proposed the related organizational factors by connecting technical competence with human context a referred relative advantage, top managers support, adequate resource and benefits to organizational context. Hsu, Ray, and Li-Hsieh [33], expanded the TOE framework, not only to explain the adoption decision appropriately but also the modalities of adoption (pricing and deployment) offered by cloud platforms. The assessment of government organization readiness required redefining the organizational dimension of TOE according to organization nature and features. Therefore, this study redefined the organizational readiness of government agencies through top management support, organizational capability and government policy. Ratten [41,42] conducted two cross-cultural comparisons in context of adoption studies based on behavioral intention of individual users, where China compared with USA and Turkey with USA respectively. The studies of CC based on the economic sector $[34,38,43]$, investigated the decision of adoption in medical industry and [10] have focused on manufacturing and services sectors. In regard to organization size, the authors in $[16,35,44]$ focused on small and medium size enterprises. These studies main 
focus was on CC success factors and organizational capabilities [11,45,46]. Sangeeta and Bharadwa [29], investigated in the impact of CC on the organizational flexibility. However there are a few empirical studies conducted in public sector, like [8] investigated in the challenges and issues influencing the adoption of CC in Australian regional municipal governments. Shin [47] examined the acceptance of CC services in government agencies by focusing on the key characteristics that affect behavioral intent based on South Korea public employees. The most important CC contribution for the public sector is sharing capacity of IT resources among multiple agencies [47]. General lack of institutional readiness is one the barriers for adoption cc in Ghanaian government agencies [48]. Liang et al [30] suggested a holistic approach by using grounded theory to examine determinants of e-government cloud adoption among government agencies in China. The studies that focused on middle east region, [49,50,51], explored CC benefits and challenges for e-government theoretically in Jordan and United Arab Emirates respectively. Other studies assessed CC adoption for e-government implementation in Yemen [40,52] and [37] investigated the factors affecting Saudi government to adopt CC. Obviously, the empirical studies investigated in adoption CC for government organizations still rarely. More attentions are needed to measure government organizations' readiness for CC.

\subsection{Research Model}

The TOE framework is the most frequently used to define factors of CC adoption. The TOE framework has a solid theoretical basis, consistent empirical support, and the potential of application to IS adoption [28]. Therefore, TOE framework is capable of providing insights for researchers and practitioners to understand the adoption of innovation in organizations [27]. This study is limited to organizational attributes of government agencies. The research model reflects organizational aspects of internal environment such as size, management support and organizational capability. The government policy support included within organizational context because it is seen as apart from government body not considered as an external environmental factor. This viewpoint is similar in their investigation about factors influencing local government decision-makers while adopting integration technologies [53]. Therefore, this study is limited to organizational dimension because the goal is to evaluate the readiness of government organizations, so the technological and environmental contexts have been excluded from research model. Government organizations readiness reflects the internal maturity and feasibility (resources, managerial and institutional) of adopting CC [30]. Therefore, the main hypothesis (H1) "the formative factors of organizational readiness (top management support, organizational capability and government policy) have a positive influence on the government agencies intentions' to adopt CC technology.”

Top Management Support (TopM): This means commitment to allocate time, resources, and encouragement to develop and use information technology applications [54]. Entrepreneurial leadership positively influenced subordinates' innovative behavior in government organizations
[55]. Top managers play an important role because CC implementation may involve integration of resources and reengineering of processes [36]. They have the ability to make the change and execute acceptance of the cloud. The IT competence of Top management and capability of providing suitable organizational environment for adopting $\mathrm{CC}$ in terms of budget, adequate human and IT resources, and time is a cornerstone to CCA [56]. Where the studies in different industries confirmed this pivotal role for top managers [10,34,35,36]. In other hand, [57] reported that lack of top management support is one of the main drawbacks of ACC by government agencies. Another opinion, that top management support is no longer a driver as organizations are starting to adopt CC services on the basis of its' advantages to support business growth [32]. It seems very important to investigate the attitude of governments leaders toward change to adopt CC.

Organizational Capability (OrgCap): There are two indicators to measure technological readiness. First, IT infrastructure readiness that refers to installed network technologies and enterprise systems, which provide a platform on which the CC applications can be built $[10,36,37]$. Technological competency is the second indicator that means the knowledge and skills possessed by IT staff to implement cloud-computing-related IT applications [34,58]. These two indicators could enhance the technological readiness of organization to adopt CC [10]. Lack of sufficient technological infrastructure and employees' knowledge about integration technologies have restricted the decision of adoption among local government agencies in U.K [53]. At other side, the sufficient and reliable resources to support the use of CC as well as appropriate learning routines and performance measures is argued to enable the adoption of CC [10,31,32,59]. Therefore, organization capability behooves to be considered in this study.

Government Policy (GovPolicy): There are many law and policy issues raised with CC that could become a problematic for government agencies, both as cloud users and as cloud providers [7]. CC policy raises important issues, which include issues of privacy, security, anonymity, telecommunications capacity, government surveillance, reliability, and liability [60]. The formation of IT policy within the government domain should assign accountability frame within the IT organization and government CIOs should be capable of governing and controlling items within their agencies and departments [7]. The key challenge presented by CC is the difficulty of fully managing and controlling cloud providers outside the government. In this case, the strong service agreements become a necessary matter. Regarding to cloud-computing as a transboundary technology, the legal support is deemed important to protect organizations that adopt CC as laws vary from country to another [31]. Organizations need to verify that the cloud provider respects the regulatory and compliance requirements especially in critical domains such as government, finance, and healthcare [59]. The inconsistent data protection laws among world countries is second factor most risky to adopt CC [61]. Alshamaila et al. [35] confirmed these geo-restrictions on adoption CC. Therefore, establishing international standardized regulations could promote trans-border processing data using CC [60]. Government organizations have more concern in terms of sovereignty over national data [4]. Without economic 
incentives, evolving cloud-computing standards and government regulations may not be sufficient to overcome barriers of CC adoption [10].

Technological development rate surpasses legislative responses therefore it is not significant in developing countries [31]. The regulations of CC in the developed world, often conflict with each other, while in developing countries they are not adequate [56]. Therefore, regulatory concerns negatively affect CC adoption in public sector specially with more sensitive data [37]. Government plays dual role as customer and regulator. Kamal et al [53] found that strong pressures from the central government have had positive influences on decision of local governments for adopting integrated technology. Policy makers could become hindrances to CC adoption especially by having wrong perceptions and lack of proper understanding of the new technology [8]. Following appropriate incentives policy will increase the rate of adoption of an innovation [62]. Therefore, Australian government devised CC strategies, issued regulations and guidelines information to benefit from CC. [8]. The institutional and political barriers are one of the main factors explaining lack of e-government adoption [63]. Such barriers preventing the emergence of a structured and trustworthy decision-making process and designing policies of e-government. This factor explained formalization and centralization of DOI model. Where formalization means the degree to which an organization obligates its members by rules and procedures. The centralization is the degree to which power and control in a system are concentrated in hands of a relatively few individuals [28]. This factor explaining the characteristic of decision-making process in public sector. This study focusing on testing the ability of internal government policy of allowing to adopt CC. However, there are supportive policies that can yield more regulations to organize and protect CC use.

Organization Size: Regarding the organization size, CC solution providers can assist organizations, especially small to medium size organizations to make savings from buying, running, and maintaining their IT infrastructure [20,35,64]. Therefore, they are more flexibility to adopt CC [35]. In contrast, [10,36] saw large organizations have an advantage over small ones because they have more affordability and can take greater risks associated with innovation adoption. However, both government organizations [37] and private [10] confirmed impact organization size on CCA. Out of tune, [31,32] found organization size insignificant. Obviously, there is a conflict regarding the relationship between the organization's size and the likelihood to adopt CC, and further in-depth studies are needed to address this conflict [56]. This factor included in order to understand in-depth the impact of size on CC adoption. Therefore, we suggest examining the organization size based on different perspective from previous studies by comparing three pre-defined groups of size: small and medium and large. Thus, the last posit (H2) "There is statistically significant difference between small, medium and large organizations in terms the determinants of organizational readiness to adopt CC technology."

Finally, the CC knowledge and IT-related experience of IT specialists and IT managers could be important for adoption intentions $[40,46]$. Thus, we consider the degree of
CC knowledge and the experience in last position as a control variable for the intention of adoption in our research model.

\section{Methodology}

The PLS path model includes a hierarchical component model (HCM) to prove that first-order constructs are highly correlated, and also to prove valuable that formative indicators exhibit high levels of collinearity [65]. HCMs have two elements: the higher-order component (HOC), which captures the more abstract higher-order construct, and the lower-order components (LOCs), which capture the subdimensions of the higher-order construct. The government policy support included in organizational factors because it seen as apart from government body not considered as an external environmental. Therefore, the research model consists of LOCs (TopM, OrgCap and GovPolicy) and HOC (organizational readiness) that encompass all LOCs factors affecting the government agencies intentions to adopt CC technology.

\subsection{Measurements and Data}

The study instruments developed in order to evaluate the theoretical model of government agencies readiness in Jordan to adopt CC. First, we had run a pilot study with a panel of IT experts from public sector and IT professors to gauge the study instrument. The instrument has been modified in accordance with suggested comments. Second, we sent a web-based survey into targeted respondents, those whom work in IT departments at variety of government organizations. The questionnaire is bilingual, Arabic and English because the study was administered in Jordan. Each statement companied with Arabic explanation that translated from English which has been reviewed by a group of IT researchers with experience about English concepts of IT. The constructs (top management, technological capability and government policy) were measured using a seven-point Likert scale on an interval level ranging from "strongly disagree" to "strongly agree" and cloud-computing adoption as a dependent construct (see Appendix A). Also, we recoded the control variables of experience and cloud knowledge by values ranging from 1 for those with minimal experience and minimal knowledge to 4 for those with higher experience and knowledge (see Table 3).

In order to define organization size more precisely, we asked respondents to talk about six indicators (see Table 2) that could scale their organization size, where adapted from $[33,46,64]$. Therefore, the mediation value of scores of all answers for each respondent has been used to define the organization size. The organizations can be categorized to three classes either small, medium or large. Thus, in order to predict the organization size, we have to find the class size using the following two equations:

$$
\text { Range }=\text { max score }-\min \text { score }
$$

Class interval $=$ range $\div$ classes number

Class interval $=(5-1) \div(3)=1.333$ 
Table 2. Organization Size

\begin{tabular}{|c|c|c|c|c|c|c|}
\hline $\begin{array}{c}\text { AVG Website } \\
\text { Visitors Yearly }\end{array}$ & Total of PCs & $\begin{array}{c}\text { Total of } \\
\text { Servers }\end{array}$ & $\begin{array}{c}\text { Total of IT Budget } \\
\text { Yearly by US\$ }\end{array}$ & $\begin{array}{c}\text { Organization } \\
\text { Branches }\end{array}$ & $\begin{array}{c}\text { Total of } \\
\text { Employees }\end{array}$ & $\begin{array}{c}\text { Response } \\
\text { Score (1 to 5) }\end{array}$ \\
\hline $1-79,999$ & $1-200$ & $1-5$ & less than 70500 & $1-5$ & $1-200$ & 1 \\
\hline $80,000-159,999$ & $201-400$ & $6-10$ & above $70500-141,000$ & $6-10$ & $201-400$ & 2 \\
\hline $160,000-239,999$ & $401-600$ & $11-15$ & above $141,000-211,500$ & $11-15$ & $401-600$ & 3 \\
\hline $240,000-319,999$ & $601-800$ & $16-20$ & above $211,500-282,000$ & $16-20$ & $601-800$ & 4 \\
\hline 320,0000 and above & Above 800 & above 20 & above 282,000 & Over 20 & Over 800 & 5 \\
\hline
\end{tabular}

Table 3. Sample Characteristics

\begin{tabular}{|c|c|c|c|c|c|c|c|c|}
\hline \multicolumn{2}{|l|}{ Respondents' Positions } & \multicolumn{2}{|c|}{ Cloud Knowledge } & \multicolumn{2}{|c|}{ Experience } & \multicolumn{3}{|c|}{ Organization Size } \\
\hline IT managers \& head of IT Div. & 59 & Excellent & 13 & 1 to 4 & 19 & Small & 77 & $58 \%$ \\
\hline Network administrator & 24 & Very Good & 45 & 5 to 10 & 43 & Medium & 25 & $19 \%$ \\
\hline Programmer & 19 & Good & 55 & 11 to 15 & 29 & \multirow{2}{*}{ Large } & \multirow{2}{*}{30} & \multirow{2}{*}{$23 \%$} \\
\hline Computer \& System Engineer & 8 & Not too much & 19 & Over 15 years & 43 & & & \\
\hline Database Administrator & 5 & \multirow{3}{*}{\multicolumn{7}{|c|}{$\begin{array}{l}\text { The new definition of organization size represents the computing power of organization based in the } \\
\text { factors shown in Table } 2 \text {. In fact, some organizations have a few employees but the AVG website visitors } \\
\text { very huge and they have bigger datacenter than large traditional institutions. Therefore, the new criteria } \\
\text { scaling organization size more precisely and objective. }\end{array}$}} \\
\hline Quality System Specialist & 8 & & & & & & & \\
\hline IT Teacher & 9 & & & & & & & \\
\hline Sample Total & \multicolumn{8}{|l|}{132} \\
\hline
\end{tabular}

Based on mediation resulted from all answers of a single respondent for size indicators, the organization could be: Small, if mediation $\leq 2.33$; Medium, if $2.33<$ mediation $\leq 3.66$; or Large organization, if mediation $>3.66$. The mediation calculated after the average computation to have more accurate score for organization size.

An online version of the questionnaire was emailed to IT managers, Heads of IT and E-gov divisions and other respondents from varied of IT disciplinary. Data were collected using an online questionnaire from March 2017 to March 2018 emailed to respondents' formal outlook email. Moreover, we have visited most of IT departments across the country in order to encourage respondents and explain the importance of the study. The respondents are qualified which indicating a good quality of data, 36.8\% of respondents' hold graduate degree in IT discipline. Around half of respondents are responsible about IT strategic planning for their organizations because their duties as IT managers and head of E-gov divisions. Although the concept of CC still new for developing countries, $85.7 \%$ of respondents have enough knowledge about CC and above. In term of respondents' experience $87 \%$ of them have more than 4 years' experience in IT field. The web-based questionnaire helps to control the quality of responses where the questions displayed randomly from one respondent to another. The option of required question activated which does not allowed to submit any uncomplete response.

\section{Results}

Partial Least Square Structural-Equation Modelling (PLS-SEM) was used to empirically assess the research model. PLS-SEM has advantages over linear regression models that latent variables are theoretical constructs could not be measured directly (such as beliefs, intentions, and feelings); they could only be measured indirectly through those characteristics we attribute to them [66]. PLS-SEM is suitable to assess both reflective and formative constructs and suited for theory development which is consistent with the study goal. We used the repeated indicator approach for reflective-formative hierarchical latent variables. This approach produces generally less biased, and therefore, more precise parameter estimates and a more reliable higher-order construct score [67]. The total respondents are (132); thus, the minimum sample size achieved both of the ten times conditions for using partial least square (PLS): (a) ten times the largest number of formative indicators used to measure one construct; or (b) ten times the largest number of structural paths directed at a particular latent construct in the structural model [65].

\subsection{Validity and Reliability}

The result of validity and reliability based on convergent validity and composite reliability. The model will be reliable if all the composite reliability (CR) values equal or greater than (0.7) [68]. All CR values for complete sample and subgroups are greater than 0.7 (see Table 4). Therefore, we can conclude the model is reliable. all of average variance extracted (AVE) values are greater than 0.50; thus, the model achieved sufficient degree of convergent validity requirements [65]. GovP4 and TopM5 items were eliminated from all groups because the loading factor less than 0.7. All other items with loadings greater than 0.7 are retained for both the full sample and all subgroups. The discriminant validity based on FornellLarcker criterion established if the square root of each construct's AVE is greater than all its correlations with other constructs in the same model [65].

Table 4. Validity and Reliability

\begin{tabular}{|c|c|c|c|c|c|c|c|c|}
\hline Organization size & \multicolumn{2}{|c|}{ All groups } & \multicolumn{2}{|c|}{ Large } & \multicolumn{2}{c|}{ Medium } & \multicolumn{2}{c|}{ Small } \\
\hline Factors & CR & AVE & CR & AVE & CR & AVE & CR & AVE \\
\hline Adoption & .91 & .72 & .96 & .85 & .89 & .67 & .89 & .68 \\
\hline GovPolicy & .86 & .67 & .89 & .72 & .87 & .70 & .85 & .66 \\
\hline OrgCap & .92 & .64 & .92 & .64 & .91 & .64 & .92 & .65 \\
\hline TopM & .90 & .70 & .90 & .69 & .91 & .72 & .91 & .71 \\
\hline
\end{tabular}


As shown in Table 5, AVE value of each construct is greater than its correlations with other constructs; thus, discriminant validity has been established. Overall, the study model passed all measures of internal consistency reliability and indicator reliability, convergent validity and finally discriminant validity. This approved the results of this study are valid and reliable.

Table 5. Discriminant Validity

\begin{tabular}{|l|c|c|c|c|}
\hline Factors & Adoption & GovPolicy & OrgCap & TopM \\
\hline Adoption & .83 & & & \\
\hline GovPolicy & .37 & .81 & & \\
\hline OrgCap & .28 & .51 & .80 & \\
\hline TopM & .53 & .46 & .51 &. $\mathbf{8 4}$ \\
\hline
\end{tabular}

\subsection{Hypotheses Testing}

Bootstrapping is a nonparametric procedure that allows testing the statistical significance of various PLS-SEM results such as path coefficients, $\mathrm{R}^{2}$ values, Indirect Effects, Total Effects, Outer Loadings, and outer weights. The analysis of hypotheses was based on the examination of the standardized paths [65]. The path significance levels were estimated using the bootstrapping method (5000 re-samples). The following table showing the result of all hypotheses for full sample and sub-groups. For the repeated indicator approach, we use all first-order construct indicators as indicators for the second-order construct (the organizational readiness dimension). To assess the effect of the OrgR dimension on intention of adoption, we model the influence on the formative higher-order dimension (OrgR) through the first-order indicators. Hence, we include paths from OrgR to intention of adoption and assess the total effect of OrgR dimension on the adoption and the indirect effect through the first-order constructs. In order to evaluate the structural model.

First, we examined for evidence of multicollinearity among inner model constructs also we have checked the existence of common method bias. All (VIF) values of first-order and second-order model are clearly below the threshold of 5. This suggests that there are no concerns of multicollinearity. The full collinearity test is a new approach for identification of common method bias based on variance inflation factors (VIFs). Therefore, if all values of VIFs resulting from a full collinearity test are equal to or lower than 3.3, the model can be considered free of common method bias [69]. We found that all VIF values less than 3.3 for all constructs model. Henceforth, we concluded that common method bias is not existed in our data.

Second, we examined the $\mathrm{R}^{2}$ for the full sample and sub-groups. The low-order components explained the organizational readiness (OrgR) perfectly because it is a formative second-order factor. Overall, the OrgR explains $35 \%$ of government agencies intention to adopt CC. However, based on the large government agencies perspectives, OrgR explains 53\% of CC adoption decision which higher than medium (46\%) and small (35\%) agencies respectively. Obviously, the OrgR of large and medium organizations has more explanation for intentions of adoption CC than small organizations. The results of total affect declared that OrgCap (50\%) has the strongest total effect on OrgR to adopt CC, followed by TopM (42\%) and GovPolicy (28\%) respectively. The government OrgR has (56\%) of total effect on the government agencies decision of adoption. The indirect effect results articulated that OrgCap (28\%) has the strongest effect on the government agencies intentions to adopt CC technology, followed by TopM (23\%) and GovPolicy (15\%).

Third, we examined the effect size $\mathrm{f}^{2}$ assessing the contribution of exogenous constructs to the endogenous latent variables $R^{2}$ value. The results of f-square test revealed that OrgR has large contribution (0.47) for intentions of government agencies to adopt CC technology. Forth, the path coefficients demonstrate that low-order components have strong relationship with high-order construct, where OrgCap (0.503) has the highest correlation value then TopM (0.422) and GovPolicy (0.275) respectively. Consequently, OrgR has strong effect (0.555) on intentions of government agencies to adopt CC technology. Interestingly, the strongest relation between OrgR and adoption reported from large organization (0.726) and lower in medium (0.716) and small organization (0.457) respectively. Hence, the relationship of higher-order component represented all its low-order constructs with intentions of adoption CC significantly at all groups level therefore (H1) accepted.

Table 6. Research Hypotheses Testing

\begin{tabular}{|c|c|c|c|c|c|}
\hline Group & Full sample (132) & Large (30) & Medium (25) & Small (77) & \multirow{3}{*}{$\begin{array}{c}\text { Acceptance } \\
\text { (Sig. }=.05)\end{array}$} \\
\hline \multirow{2}{*}{ Paths } & Path coefficient $(\beta)$ & $\beta$ & $\beta$ & $\beta$ & \\
\hline & t-value & t-value & t-value & t-value & \\
\hline \multirow{2}{*}{ H1 OrgR-> Adoption } & .555 & .726 & .716 & .469 & \multirow{2}{*}{ Supported } \\
\hline & 7.658 & 8.214 & 2.649 & 3.847 & \\
\hline \multirow{2}{*}{ TopM-> OrgR } & .422 & .396 & .422 & .420 & \multirow{2}{*}{ Supported } \\
\hline & 11.104 & 5.704 & 6.531 & 7.373 & \\
\hline \multirow{2}{*}{ OrgCap-> OrgR } & .503 & .479 & .442 & .545 & \multirow{2}{*}{ Supported } \\
\hline & 12.188 & 4.567 & 4.861 & 12.255 & \\
\hline \multirow{2}{*}{ GovPolicy-> OrgR } & .275 & .330 & .277 & .246 & \multirow{2}{*}{ Supported } \\
\hline & 8.620 & 3.669 & 5.839 & 6.179 & \\
\hline $\mathbf{R}^{2}$ & $\begin{array}{c}\text { Adoption }=.353 \\
\text { OrgR }=1\end{array}$ & $\begin{array}{c}\text { Adoption }=.505 \\
\text { OrgR }=1\end{array}$ & $\begin{array}{c}\text { Adoption= }=.411 \\
\text { OrgR }=1\end{array}$ & $\begin{array}{c}\text { Adoption }=.22 \\
\text { OrgR }=1\end{array}$ & \\
\hline
\end{tabular}

Note: OrgR r-square $=1$, because OrgR a second-order formative factor. 


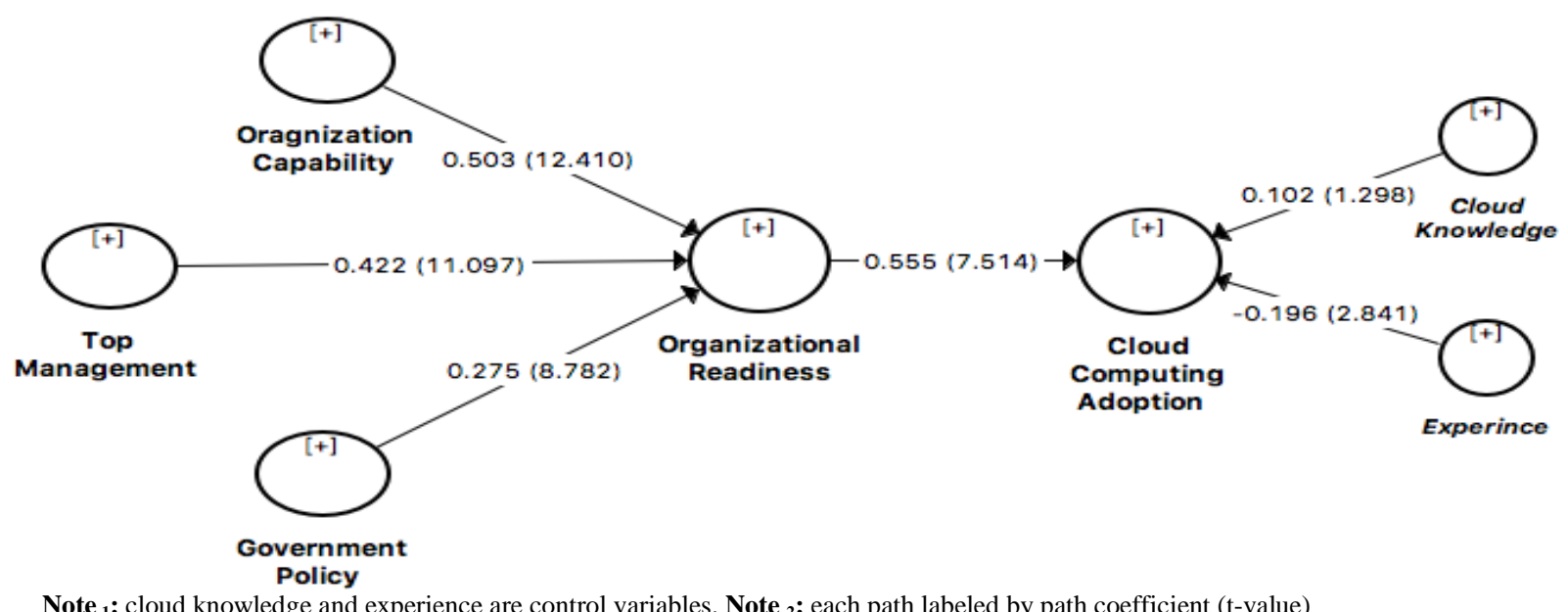

Figure 1. Structural model of organizational readiness

Table 7. Control variables testing

\begin{tabular}{|c|c|c|c|c|}
\hline Group & $\begin{array}{c}\text { Full sample } \\
(132)\end{array}$ & $\begin{array}{c}\text { IT managers } \\
(58)\end{array}$ & $\begin{array}{c}\text { Other IT specialists } \\
\text { (74) }\end{array}$ & \multirow{3}{*}{$\begin{array}{l}\text { Acceptance } \\
\text { (Sig.=.05) }\end{array}$} \\
\hline \multirow{2}{*}{ Paths } & Path coefficient $(\beta)$ & $\beta$ & $\beta$ & \\
\hline & t-value & t-value & t-value & \\
\hline \multirow{2}{*}{ Cloud knowledge -> Adoption } & .102 & .096 & .089 & \multirow{2}{*}{ Not Supported } \\
\hline & 1.290 & 1.174 & .746 & \\
\hline Experience -> Adoption & -.196 & -.267 & -.143 & Partial supportec \\
\hline
\end{tabular}

As shown in Table 6, the path coefficients for the three groups are numerically different, but the question is whether the differences are statistically significant. The multi-group analysis applied to test if pre-defined groups (large, medium and small organizations) have significant differences in their group-specific parameter estimates (e.g., outer weights, outer loadings and path coefficients) [70]. Consequently, testing hypothesis (H2), if sub-groups of organization size have significance difference in terms the determinants of organizational readiness (top management support, government policy, and organization capability) to adopt CC technology. A result is significant at the 5\% probability of error level, if the p-value is smaller than 0.05 or larger than 0.95 for a certain difference of group-specific path coefficients. The result of multi-group analysis for path coefficient revealed there are no any p-value less than 0.05. This indicated there are no statistical difference among the large, medium and small government agencies in regard to organizational dimension to adopt CC. Thus, the hypothesis (H2) of moderator factor which is organization size rejected.

Regarding to control variables (cloud knowledge and the experience of IT managers and IT specialists), only experience has a significant negative effect $(-0.196)$ on the intention of adoption. For this purpose, we set two distinct groups IT managers and head of IT sections as one group and others IT specialists as another group (see Table 7). The result of IT managers group has more significant negative effect $(-0.267)$ while the IT specialists) group has insignificant negative effect $(-0.143)$ on the intention of adoption. This interesting result may indicate that IT managers with more experience on their positions are more unlike to adopt CC. The cloud knowledge a control variable has insignificant effects $(0.102)$ on intentions of adoption and follow-up studies need to confirm this finding.

\section{Discussion and Implications}

Our study has revealed that organizational aspects have strong influences on the intentions of government organizations to adopt CC regardless of their size. We classified the organizations based on their computing power into large, medium and small organizations. Then after applying multigroup analysis by smartPLS, we found no significant difference between large, medium and small organizations in terms of organizational readiness for adoption CC. This finding suggests that top management support, organization capability and government policy all important predictors of organizational readiness to adopt new technology such as CC for large, medium and small government agencies. This results confirmed that general lack of organizational readiness is one the barriers to adopt CC for government agencies in Ghana [48]. The study highlighted some important issues when government agencies intent to adopt CC for their own purposes.

Firstly, As the organization capability important for private sector organizations [10,31,32,36,59], this study confirmed this finding in government domain. The organization capability factor has the highest impact on organizational readiness for adoption $\mathrm{CC}$, which is suggesting that very important for organization to own technological competency and technological readiness in order to be ready for the CC age. Government organizations should have the IT skills and financial ability to be able enjoy the advantages of CC.

Secondly, the management support is a critical factor to strength organization capability, especially top managers 
are responsible to provide the required resources for adoption CC such as human capital and finical resources. The enthusiasm of top managers and their ability to bear the risks of $\mathrm{CC}$ will release the required resources for adoption CC. The results demonstrate the importance of the contribution top managers to organizational readiness to adopt CC. Thus, the awareness of top managers for CC features and risks is very important to embrace CC advantages successfully. The results indicated to the importance role of top management support in advancing the organizational readiness to have sophisticated technology.

Thirdly, if existed government policy supported the organizational readiness for adoption CC. The decisionmakers of government agencies will support the decision of adoption and will allocate more resources for the adoption process based on supportive government policy. Consequently, the internal policy of government agencies should be consistent with CC services. However, without a robust and active policy to support usage of CC, government agencies will lack the legal framework to accept this new model. The supportive policy is very important where the studies mentioned that lack of policy support one of the key barriers for a successful e-government implementation [63,71]. In order to prevent extension these barriers into CC, the findings suggest government agencies to revise their internal policy and the policy of government overall in order to be consistent with the new IT resources model. The new policy should encompass a suitable purchasing mechanism instead the traditional government purchasing procedures. In traditional IT resources, government agencies storing and processing data in their own servers but with CC concept the data will be processed and stored in elsewhere owned by cloud service providers or by another government entity working as operator and intermediate between cloud providers and other government agencies. As such the government policy should clearly identify the roles and responsibilities each party toward government data. The service level agreement is supposed to be aligned with government policy. Consequently, government should possess the enough expertise to establish a robust and comprehensive contract with CC service providers. Government agencies responsible building the internal competency and accommodating the thoughts of CC service providers and other partners. The control variables revealed that degree of knowledge of CC is insignificant to the government agencies' intentions to adopt CC, and this similar to finding reported by [40] from government domain in Yemen. This could be referred that governments of developing countries usually take the decision of adoption based on consultation of external experts. Another interesting result is that if IT managers and IT specialists have more IT-related experience, they are less likely to intention of adoption. Specially, the experience of top IT managers has more negative significant effect than other IT specialists. This referred to loyalty of IT managers to the traditional IT model. Also, IT managers may experience were limited to the obsolete technology; therefore, they resist to shift to cutting-edge technology which they have less experience with it. Overall, the model could help the decision-makers of government agencies to evaluate their organizational readiness for CC adoption. The model provides viable tool to formulate the change management strategy for CC adoption.

\section{Conclusion}

This study sought to measure the government sector readiness to adopt CC. This study has been more precise on organizational aspects of the government sector instead the multidimensional models in previous studies that tackled private sector organizations. The research model developed based on TOE framework and empirically tested by IT managers and IT specialists across government sector organizations. This study participated with a few previous studies to quest the determinants of CC adoption in public sector. Also, the study shed the light on new IT services model to overcome technological and IT infrastructures barriers in developing countries such Jordan. The results indicated that organization capability, top management support and government policy are crucial indicators of organization readiness for CC adoption. Our study also found that large, medium and small organizations have similar drivers of CC adoption, which underscores the importance of considering the organizational characteristics of government agencies across different organizational levels.

The HCM examined the readiness of government agencies to adopt CC based on manifestation of underlying factors. The top managers support is substantive to augment organization capability both IT infrastructure readiness and technological competency. Moreover, the supportive policy is essential for decision-makers of government organizations to provision the required resources to adopt CC. Thus, the new structure for organizational context of TOE successfully established.

The assessment of CC adoption based on scientific approach that takes into consideration organizational characteristics is more meaningful and feasible to provide valuable insight for practitioners and researchers. Overall, this study contributes to understand the drivers of organizational readiness for adoption of a novel CC for government agencies and enriches both academic literatures of government adoption and as a reference for practitioners to develop government policies and adoption strategy. However, the sample size is one of limitations for this study where some sub-groups number of respondents less than thirty. We encourage researchers to apply the model to measure organization readiness with bigger sample size and compare different industries and different organizations size. We recommend to researchers apply our methodology to define the organization size which could help to improve it and confirm the validity of our contribution. Also, more investigations needed for the control variables like experience and degree of CC knowledge on the intention of adoption. since our study and other previous studies mainly adopted TOE, we suggest for researchers to consider Technology Acceptance Model (TAM), Theory of Reasoned Action (TRA) and Theory of Planned Behavior (TPB) in order to assess adoption of CC services. As well as, the holistic approach and in-depth study for the technological determinants of adoption CC is needed to evaluate the acting of government agencies with this new technology. 


\section{References}

[1] M. Armbrust et al., "A View of Cloud Computing," J. Comput. Cult. Herit., vol. 53, no. 4, pp. 50-58, 2010.

[2] A. Prasad, P. Green, and J. Heales, "On governance structures for the cloud computing services and assessing their effectiveness," Int. J. Account. Inf. Syst., vol. 15, no. 4, pp. 335-356, 2014.

[3] F. Liu et al., "NIST Cloud Computing Reference Architecture Recommendations of the National Institute of Standards and," 2011.

[4] K. Irion, "Government cloud computing and national data sovereignty,” Policy and Internet, vol. 4, no. 3-4, pp. 40-71, 2012.

[5] D. C. Chou, "Cloud computing: A value creation model," Comput. Stand. Interfaces, vol. 38, no. 2015, pp. 72-77, 2015.

[6] S. Greengard, "Cloud computing and developing nations," Commun. ACM, vol. 53, no. 5, pp. 18-20, 2010.

[7] S. Paquette, P. T. Jaeger, and S. C. Wilson, "Identifying the security risks associated with governmental use of cloud computing,” Gov. Inf. Q., vol. 27, no. 3, pp. 245-253, 2010.

[8] O. Ali, J. Soar, and J. Yong, "An investigation of the challenges and issues influencing the adoption of cloud computing in Australian regional municipal governments,” J. Inf. Secur. Appl., vol. 27-28, pp. 19-34, 2016.

[9] A. S. M. Al-rawahna, S.-C. Chen, and C.-W. Hung, "The Barriers of E-Government Success: An Empirical Study from Jordan," Int. J. Manag. Public Sect. Inf. Commun. Technol., vol. 9, no. 2 pp. 1-18, 2018.

[10] T. Oliveira, M. Thomas, and M. Espadanal, "Assessing the determinants of cloud computing adoption: An analysis of the manufacturing and services sectors," Inf. Manag., vol. 51, no. 5, pp. 497-510, 2014.

[11] G. Garrison, S. Kim, and R. L. Wakefiel, "Success Factors for Deploying Cloud Computing," Commun. ACM, vol. 55, no. 9, pp. 62-68, 2012

[12] D. C. Wyld, "Moving to the Cloud: An Introduction to Cloud Computing in Government E-Government," Washington, DC 20005, 2009

[13] T. Fredriksson et al., "Information Economy report 2013: The Cloud Economy and Developing Countries,” UNITED NATIONS, New York and Geneva, 2013.

[14] R. Martins, T. Oliveira, and M. A. Thomas, "An empirical analysis to assess the determinants of SaaS diffusion in firms," Comput. Human Behav., vol. 62, pp. 19-33, 2016.

[15] S. Subashini and V. Kavitha, "A survey on security issues in service delivery models of cloud computing," J. Netw. Comput. Appl., vol. 34, no. 1, pp. 1-11, 2011.

[16] P. Gupta, A. Seetharaman, and J. R. Raj, "The usage and adoption of cloud computing by small and medium businesses," Int. J. Inf Manage., vol. 33, no. 5, pp. 861-874, 2013.

[17] X. Yang, B. Nasser, M. Surridge, and S. Middleton, "A businessoriented Cloud federation model for real-time applications,” Futur. Gener. Comput. Syst., vol. 28, no. 8, pp. 1158-1167, 2012.

[18] S. Marston, Z. Li, S. Bandyopadhyay, J. Zhang, and A. Ghalsasi, "Cloud computing - The business perspective," Decis. Support Syst., vol. 51, no. 1, pp. 176-189, 2011.

[19] R. Dukaric and M. B. Juric, "Towards a unified taxonomy and architecture of cloud frameworks,” Futur. Gener. Comput. Syst., vol. 29, no. 5, pp. 1196-1210, 2013.

[20] A. Lin and N. C. Chen, "Cloud computing as an innovation: Percepetion, attitude, and adoption,” Int. J. Inf. Manage., vol. 32, no. 6, pp. 533-540, 2012.

[21] D. Zissis and D. Lekkas, "Addressing cloud computing security issues,” Futur. Gener. Comput. Syst., vol. 28, no. 3, pp. 583-592, 2012.

[22] J. S. Ward and A. Barker, "Observing the clouds: a survey and taxonomy of cloud monitoring," J. Cloud Comput. Adv. Syst. Appl., vol. 3, no. 24, pp. 1-30, 2014

[23] D. Zissis and D. Lekkas, "Securing e-Government and e-Voting with an open cloud computing architecture," Gov. Inf. Q., vol. 28, no. 2, pp. 239-251, 2011.

[24] D. C. Wyld, "The Cloudy Future Of Government IT: Cloud Computing and The Public Sector Around The World," Int. J. Web Semant. Technol., vol. 1, no. 1, pp. 164-169, 2010.

[25] J. A. González-Martínez, M. L. Bote-Lorenzo, E. Gómez-Sánchez, and R. Cano-Parra, "Cloud computing and education: A state-ofthe-art survey,” Comput. Educ., vol. 80, pp. 132-151, 2015.
[26] R. Balasubramanian, "Security Issues: Public vs Private vs Hybrid Cloud Computing,” Int. J. Comput. Appl., vol. 55, no. 13, pp. 35-41, 2012.

[27] Y. K. Dwivedi, M. R. Wade, and S. L. Schneberger, Information Systems Theory, vol. 28. 2012

[28] T. Oliveira and M. Martins, "Literature review of information technology adoption models at firm level," Electron. J. Inf. Syst. Eval., vol. 14, no. 1, pp. 110-121, 2011.

[29] P. L. Sangeeta and S. Bharadwa, "Understanding the impact of cloud-based services adoption on organizational flexibility: An exploratory study,” J. Enterp. Inf. Manag., vol. 29, no. 4, pp. 566-588, 2016.

[30] Y. Liang, G. Qi, K. Wei, and J. Chen, "Exploring the determinant and influence mechanism of e-Government cloud adoption in government agencies in China," Gov. Inf. Q., vol. 34, no. 3, pp. 481-495, 2017.

[31] E. Senyo, P. K., Effah, J., \& Addae, "Preliminary insight into cloud computing adoption in a developing country," J. Enterp. Inf. Manag., vol. 29, no. 4, pp. 505-524, 2016.

[32] A. Gutierrez, E. Boukrami, and R. Lumsden, "Technological, organisational and environmental factors influencing managers' decision to adopt cloud computing in the UK," J. Enterp. Inf. Manag., vol. 28, no. 6, pp. 788-807, 2015

[33] P. F. Hsu, S. Ray, and Y. Y. Li-Hsieh, "Examining cloud computing adoption intention, pricing mechanism, and deployment model," Int. J. Inf. Manage., vol. 34, no. 4, pp. 474-488, 2014.

[34] J. W. Lian, D. C. Yen, and Y. T. Wang, “An exploratory study to understand the critical factors affecting the decision to adopt cloud computing in Taiwan hospital,” Int. J. Inf. Manage., vol. 34, no. 1, pp. 28-36, 2014

[35] Y. Alshamaila, S. Papagiannidis, and F. Li, "Cloud computing adoption by SMEs in the north east of England: A multiperspective framework," J. Enterp. Inf. Manag., vol. 26, no. 3, pp. 250-275, 2013.

[36] C. Low, Y. Chen, and M. Wu, "Understanding the determinants of cloud computing adoption,” Ind. Manag. Data Syst., vol. 111, no. 7, pp. 1006-1023, 2011.

[37] M. Alsanea and J. Barth, "Factors Affecting the Adoption of Cloud Computing in the Government Sector: A Case Study of Saudi Arabia," Int. J. Cloud Comput. Serv. Sci., vol. 3, no. 6, pp. 1-16, 2014.

[38] O. Harfoushi, A. H. Akhorshaideh, N. Aqqad, and M. Al Janini, "Factors Affecting the Intention of Adopting Cloud Computing in Jordanian Hospitals," Commun. Netw., vol. 8, pp. 88-101, 2016.

[39] S. Das and M. Dayal, "Exploring determinants of cloud-based enterprise resource planning ( ERP ) selection and adoption: A qualitative study in the Indian education sector," J. Inf. Technol. Case Appl. Res., vol. 18, no. 1, pp. 11-36, 2016.

[40] F. Mohammed, O. Ibrahim, and N. Ithnin, "Factors influencing cloud computing adoption for e-government implementation in developing countries Instrument development," J. Syst. Inf. Technol., vol. 18, no. 3, pp. 297-327, 2016.

[41] V. Ratten, "Factors influencing consumer purchase intention of cloud computing in the United States and Turkey: The role of performance expectancy, ethical awareness and consumer innovation Vanessa," EuroMed J. Business, vol. 10, no. 1, pp. 80-97, 2015.

[42] V. Ratten, "A US-China comparative study of cloud computing adoption behavior: The role of consumer innovativeness, performance expectations and social influence Vanessa," J. Entrep. Emerg. Econ., vol. 6, no. 1, pp. 53-71, 2014.

[43] P. J. Hsieh and W. Sen Lin, "Explaining resistance to system usage in the PharmaCloud: A view of the dual-factor model," Inf. Manag., vol. 55, no. 1, pp. 51-63, 2018.

[44] N. A. Sultan, "Reaching for the 'cloud': How SMEs can manage," Int. J. Inf. Manage., vol. 31, no. 3, pp. 272-278, 2011.

[45] G. Garrison, R. L. Wakefield, and S. Kim, "The effects of IT capabilities and delivery model on cloud computing success and firm performance for cloud supported processes and operations," Int. J. Inf. Manage., vol. 35, no. 4, pp. 377-393, 2015.

[46] C. M. Messerschmidt and O. Hinz, "Explaining the adoption of grid computing: An integrated institutional theory and organizational capability approach,” J. Strateg. Inf. Syst., vol. 22, no. 2, pp. 137-156, 2013.

[47] D. H. Shin, "User centric cloud service model in public sectors: Policy implications of cloud services," Gov. Inf. Q., vol. 30, pp. 194-203, 2013. 
[48] S. Tweneboah-Koduah, B. Endicott-Popovsky, and A. Tsetse, "Barriers to Government Cloud Adoption," Int. J. Manag. Inf. Technol., vol. 6, no. 3, pp. 1-16, 2014.

[49] I. Elbadawi, "Cloud computing for e-government in UAE: opportunities, challenges and service models," in Proceedings of the 5th International Conference on ..., 2011, pp. 1-2.

[50] T. Almarabeh, Y. K. Majdalawi, and H. Mohammad, "Cloud Computing of E-Government,” Commun. Netw., vol. 8, pp. 1-8, 2016.

[51] S. Alsharafat, B. M. Alfawwaz, and A. M. Al-shatnawi, "Jordanian Cloud-Government between Implementation and Challenges,” Int. J. Comput. Appl., vol. 102, no. 5, pp. 10-15, 2014.

[52] F. Mohammed, O. Ibrahim, M. Nilashi, and E. Alzurqa, "Cloud computing adoption model for e-government implementation," Inf. Dev., vol. 33, no. 3, pp. 303-323, 2017.

[53] M. M. Kamal, A. Z. Bigdeli, M. Themistocleous, and V. Morabito, "Investigating factors influencing local government decision makers while adopting integration technologies (IntTech)," Inf. Manag., vol. 52, no. 2, pp. 135-150, 2014.

[54] S. Petter, W. DeLone, and E. R. McLean, "Information systems success: The quest for the independent variables," J. Manag. Inf. Syst., vol. 29, no. 4, pp. 7-62, 2013.

[55] Q. Miao, G. Schwarz, A. Newman, and B. Cooper, "How Leadership and Public Service Motivation Enhance Innovative Behavior," Public Adm. Rev., vol. 78, no. 1, pp. 71-81, 2017.

[56] R. F. El-Gazzar, "A Literature Review on Cloud Computing Adoption Issues in Enterprises," in Creating Value For All Through It, First., B. Bergvall-Kåreborn and P. A. Nielsen, Eds. Springer Berlin Heidelberg, 2014, pp. 214-242.

[57] S. Yousef, "Cloud Computing in the Palestinian Public Sector, Opportunities and Challenges,” An-Najah National University, 2015.

[58] H. Sallehudin, R. C. Razak, and M. Ismail, "Factors Influencing Cloud Computing Adoption in the Public Sector: An Empirical Analysis,” J. Entrep. Bus., vol. 3, no. 1, pp. 30-45, 2015.

[59] N. Phaphoom, X. Wang, S. Samuel, S. Helmer, and P. Abrahamsson, "A survey study on major technical barriers affecting the decision to adopt cloud services,” J. Syst. Softw., vol. 103, pp. 167-181, 2015.

[60] P. T. Jaeger, J. Lin, and J. M. Grimes, "Cloud computing and information policy: Computing in a policy cloud?,” J. Inf. Technol. Polit., vol. 5, no. 3, pp. 269-283, 2008.

[61] A. Dutta, G. C. A. Peng, and A. Choudray, "Risks in Enterprise Cloud Computing: The Perspective of IT Experts," J. Comput. Inf. Syst., vol. 53, no. 4, 2013.

[62] E. M. Rogers, Diffusion of Innovations, 4th ed. US: Simon \& Schuster Inc., 1995.

[63] A. Savoldelli, C. Codagnone, and G. Misuraca, "Understanding the e-government paradox: Learning from literature and practice on barriers to adoption," Gov. Inf. Q., vol. 31, pp. S63-S71, 2014.

[64] S. C. Misra and A. Mondal, "Identification of a company's suitability for the adoption of cloud computing and modelling its corresponding Return on Investment,” Math. Comput. Model., vol. 53, no. 3-4, pp. 504-521, 2011.

[65] J. F. Hair jr, G. T. M. Hult, C. M. Ringle, and M. Sarstedt, A Primer on Partial Least Squares Structural Equation Modeling PLS-SEM), First. Los Angeles, CA: Sage Publications, Inc., 2014.

[66] D. Gefen, E. E. Rigdon, and D. Straub, "An Update and Extension to SEM Guidelines for Administrative and Social Science Research.,” MIS Q., vol. 35, no. 2, p. iii-A7, 2011.

[67] J. M. Becker, K. Klein, and M. Wetzels, "Hierarchical Latent Variable Models in PLS-SEM: Guidelines for Using ReflectiveFormative Type Models,” Long Range Plann., 2012.

[68] J. F. Hair Jr, M. Sarstedt, L. Hopkins, and V. G. Kuppelwieser, "Partial least squares structural equation modeling (PLS-SEM)," Eur. Bus. Rev., vol. 26, no. 2, pp. 106-121, 2014.

[69] N. Kock, "Common method bias in PLS-SEM: A full collinearity assessment approach,” Int. J. e-Collaboration, vol. 11, no. 4, pp. 1-10, 2015.

[70] M. Sarstedt, J. Henseler, and C. M. Ringle, "Multigroup Analysis in Partial Least Squares (PLS) Path Modeling: Alternative Methods and Empirical Results," Int. Mark. Adv. Int. Mark., vol. 22, no. January, pp. 195-218, 2011.

[71] N. P. Rana, Y. K. Dwivedi, and M. D. Williams, "Analysing challenges, barriers and CSF of egov adoption,” Transform. Gov. People, Process Policy, vol. 7, no. 2, pp. 177-198, 2013.

\section{Appendix A: Model constructs and items}

\begin{tabular}{|c|l|c|}
\hline Code & Items & Factor loading \\
\hline Adopt & Cloud-computing adoption, adopted from [10,33] & .898 \\
\hline ADOP1 & Cloud-computing adoption is useful decision & .902 \\
\hline ADOP2 & I think our organization is ready to adopt Cloud-computing & .752 \\
\hline ADOP3 & We assume to implement Cloud-computing within 2 years & .844 \\
\hline ADOP4 & We believe that most of government organizations in Jordan, like us, are interested to adopt CC & \\
\hline OrgCap & Organizational capability adopted from [10,33,34,45] & .848 \\
\hline OrgCaP1 & Our staff using IT resources in their duties & .701 \\
\hline OrgCap2 & Organization has the necessary skills to implement cloud-computing & .802 \\
\hline OrgCap3 & Our staff have Internet access, if their work requiring & .872 \\
\hline OrgCap4 & Organization knows how IT can be used to support operations & .777 \\
\hline OrgCap5 & Adequate network capacity and bandwidth & .802 \\
\hline OrgCap6 & Organization able to integrate new IT into existing infrastructure quickly & \\
\hline GovPolicy & Government policy adapted based on [10] & .898 \\
\hline GovP1 & Cloud-computing one of the government major policies & .838 \\
\hline GovP2 & Government policy not an obstacle to use cloud-computing & .712 \\
\hline GovP3 & Government intends to issue supporting regulations for cloud-computing & dropped \\
\hline GovP4 & The centralization of decision-making in government not an obstacle & \\
\hline TopM & Top management support adopted from [32,34] & .860 \\
\hline TopM1 & Enthusiasm to adopt cloud-computing & dropped \\
\hline TopM2 & To provide resources for adopting cloud-computing & .812 \\
\hline TopM3 & To understand the benefits of cloud-computing & .865 \\
\hline TopM4 & To encourage the development of cloud-computing & \\
\hline TopM5 & They lack the ability to leverage IT as a strategic core competence & \\
\hline & & \\
\hline
\end{tabular}

